The apparent size of the earth if seen from the sun would be that of a circle whose radius is the amount of the sun's parallax; or just equal to that of a sphere $3 \stackrel{8}{10}$ inches in diameter, seen from the distance of one mile.

(To be continued.)

\title{
SPECTRA OF METALLIC COMPOUNDS.
}

\author{
By Prof. A. R. LeEDs.
}

THE constant employment of the spectroscope in qualitative analysis renders it of great importance to detect and carefullly remove any attendant sources of error. Now, there are many other compounds, besides those of the alkalies and alkaline earths, which afford spectra, and a similarity in the position of their spectral linis and banda, causes them to be mistaken on oursory examination for epeetre of certin of the alkwine elements: An examination of all the more important metwllie compounds, and their faith. ful mapping, so far as they differed from other, would eliminate this source of error. Such maps, moreover, would enlarge the field of application of the spectroscope, and ensble us to detect the presence of many other bodies than those to which its use is at present restricted. A comparison of these spectra woinld at rest many interesting points of inquiry and speoulation. As for example, the amount and kind of alteration which place in the position, number and relative intensity of spectrum lines at various temperatures. For the variable influence-the temperature-being but one function of the apectrum, it is by neans to be concluded without experimental inquiry that the lese and more refrangible parts of spectra alter perri pasur. The fact that at high temperatures, decomposition takes place has already led (see memoir of M. Diacon, Ann. de Chim. [4], ir., 5) to a rariety of intereating results. He found that in cortain cases, when mixtures of volatile compounds were examined in the spectroscope, the spectrum ob. tained was not that of the compounds previously existent in the mixture, but that of the compounds which had been formed from their decomposition and subsequent recombination, according to the strength of their affinities at elevated temperatures. For example, a mixture of baryta and calcic chloride gave not only the spectra proper to these two compounds, but that of barytic chloride as well. The careful study of these changes would remove a source of em. 
barassment in spectroscopic analysis. Moreover, it would probably furnish some information upon the chemistry of compounds, which we are wont to study in the solid state or in solution, when converted into vapors, and upon the phenomena of disassociation.

We already possess a very laborious and extensive series of determinations of the spectra of compounds by Prof. A. Mitscherlich (Pogg. Ann., No. 3, 1864, and Phil. Mag. [4], September, 1864). He found that compounds of the first order, in so far as they are volatile and remain undecomposed when adequately heated, always exhibit spectra which differ completely from those of the metals. He obtained the spectra in a variety of ways. 1st. By evaporating solutions in a narrow flame of coal-gas or hydrogen. $2 \mathrm{~d}$. By bringing the substances into the flame of an oxygen-coal-gas burner. $3 d$. By bringing them into a hydrogen-chlorine burner. 4th. Evaporating bromine and iodine in hydrogen, and volatilizing the substance in the flame produced by the burning of this mixture in air or oxygen. 5th. By passing the gas to be examined either alone or in case it is not combustible, along with carbonic oxide or hydrogen, through the middle aperture of an oxyhydrogen burner, and burning the mixture in air or hydrogen. 6th. By volatilizing the substance in a current of hydrogen, and igniting the jet thus charged with the substance for examination. 7th. By passing the electric spark between electrodes of the metals or of their salts, when surrounded by an atmosphere of various gases. 8th. By using solutions of metallic salts as electrodes, and passing the spark from liquid to liquid.

It will be seen by a comparison of these methods, that they differ greatly with regard to the temperature at which the spectra are formed. It is much lower when, as in the first method, the solutions of the salts are volatilized, than when the fused salts them. selves are used. In the latter case, the spectra are much more brilliant and persistent, and the lines are more numerous.

The third method was improved by Diacon, in that he surrounded his hydrogen-chlorine burner with a hood in such a way as to prevent the vaporized substance from coming into contact with the air. He thus obtained the spectra of the chlorides unmixed with the spectra of the corresponding oxides.

The feeble illumination in the green part of the spectrum, when hydrogen is burned in chlorine, may fairly be attributed, according to the same interpretation which we apply to the spectra of tho 
metals when burned in chlorine, to the chloride of hydrogen or hydrochloric acid, and the broad bluish-green, nebulous band when hydrogen is burned in air or oxygen, to the spectrum of aqueous vapor. The spectra of metals as obtained by Mitscherlich with the electric spark have been redetermined by Huggins and Miller with great care, and the lines obtained have been referred to a scale in which the atmospheric lines form fiducial points.

The maps given by Mitscherlich and Diacon, being referred to an arbitrary scale, are intelligible with difficulty. This difficulty applies not only to the position of the lines, but in a still greater degree, to their relative intensity and brightness. It was very much to be desired that the benefits of their labors should be made available to us in the present advanced state of spectroscopy, and an attempt was made to reduce their measurements to normal wavelengths according to Angstrom's tables. But this was altogether impossible with Diacon's map, since the intervals had been micrometrically determined, and no comparison has been made with the solar or other standard lines. In Mitscherlich's maps, the lines $a, a, \mathrm{D}, \mathrm{E}, b$ and $\mathrm{F}$ are marked, but when a graphical construction was attempted, by making the values of these points, as given in the maps, the ordinates and their corresponding wave-lengths, the abscissas of a curve, the curve was so irregular that the attempt had to be abandoned.

For obtaining the spectra of the metallic compounds, I have em. ployed in the present investigation, the flame of a Bunsen burner, since it is to this source of heat that we refer our spectra in ordi. dinary laboratory work. The resulting spectra are of two different kinds. In the case of oxygen salts, we obtain the spectra of the oxide of the metallic radical, the lines and bands being more or less broadened and brightened according to the degree of volatility of the salt. With haloid salts, we obtain the spectrum proper to the compound and also the spectrum of the oxide of the metallic radi. cal. Two instruments were employed in the observations, one, a single prism spectroscope made by Desaga of Heidelberg, the other a five-prism direct-vision spectroscope made by Hofmann of Paris. Both were provided with arbitrary photographic scales. The numbers obtained in both cases were reduced to the scale accompanying the colored drawings of the metallic spectra, by Bunsen and Kirchhoff, * a dash between two numbers indicates a continuity of the spectrum between the points corresponding to them.

* $\mathrm{K} \alpha=17 \cdot 5, \mathrm{Na}=60 \cdot 4, \mathrm{Li} i=31 \cdot 8, \mathrm{C} a \alpha=42, \mathrm{Ca} \beta=60 \cdot 8, \mathrm{Sr}_{\delta}=105$. 
Table of Spectral Lines and Bands.

\begin{tabular}{|c|c|}
\hline sul & Position of Lines according to Bungen and Kirchhoff'B Mep. \\
\hline $\mathrm{s}^{2}$ & $37 \cdot 8-44 \cdot 8 \quad 60 \cdot 8-72 \cdot 4 \quad 78 \cdot 2 \quad 84 \cdot 1$ \\
\hline ater & $60 \cdot 8-72 \cdot 4$ \\
\hline $\mathrm{Ca}$ & $39 \cdot 5-43 \cdot 8-46-47 \cdot 660$ \\
\hline Ace & $34.643 .8-465860.5-62.767 .468 .9-69.47$ \\
\hline & $5-46 \quad 60 \cdot 5 \quad 67 \cdot 4 \quad 69 \cdot 8 \quad 72 \cdot 2 \quad 81.5 \quad 84-84.5$ \\
\hline & 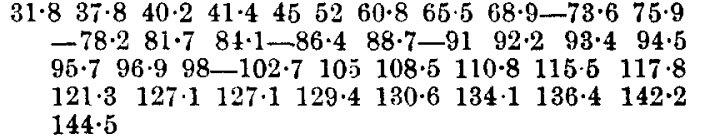 \\
\hline ................. & 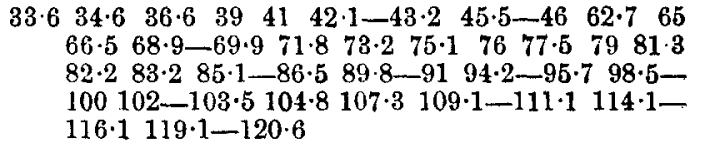 \\
\hline Plumbic Peror & $\begin{array}{c}555-58 \cdot 5 \quad 59 \cdot 5-64 \cdot 2 \quad 66 \cdot 5-68 \cdot 4 \quad 69 \cdot 9-71 \cdot 8 \quad 73 \cdot 6- \\
7680 \cdot 3-83 \cdot 2\end{array}$ \\
\hline lumbic $\mathrm{C}$ & $39-43.8 \quad 46-48.6 \quad 53-56.558 .5-59.561 .563 .766 \ldots$ \\
\hline Iumbic $\mathrm{Ni}$ & 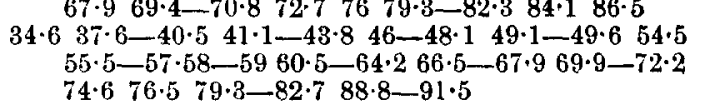 \\
\hline Plumbic Ch & 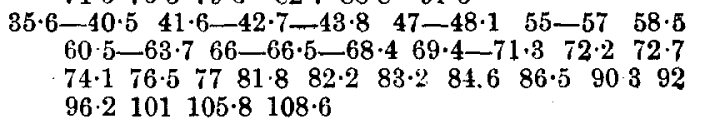 \\
\hline Fanganous Chlor & $\begin{array}{c}40 \cdot 2-42 \cdot 647 \cdot 4-49 \cdot 857-59 \cdot 4 \quad 62-64 \cdot 3-65 \cdot 5-66 \cdot 5 \\
68 \cdot 9-74 \cdot 8 \quad 75 \cdot 9-84 \cdot 1\end{array}$ \\
\hline dmic & $71 \cdot 3-72 \cdot 275 \cdot 5-78 \quad 80 \cdot 3-81 \cdot 8 \quad 88 \cdot 5-89 \cdot 8$ \\
\hline
\end{tabular}

In the accompanying map of the spectra of metallic compounds the distances on the horizontal divisions of the scale are taken as abscissas, and the relative intensities of the lines and bands as ordinates. It will be seen by comparison of the spectra of the oxygen salts of copper that there is a close similarity between them. Indeed, it is probable that if the drawing had been made directly from the instrument, instead of from notes taken of observations and used in drawing afterwards the spectra would have been almost identical. The differences would have consisted merely in the breadth and brightness of the lines. But between the spectra of the oxygen and haloid salts, and between the haloid salts themselves, the differences are numerous and striking: In addition to the lines in the less refrangible portion of the spectrum, which are common to all, and which belong to the metal as oxide, a great number of lines in the green, blue, indigo and violet are seen, whose form and grouping are peculiar to the haloid salt under examination. In the spectrum of cupric 
chloride, the most noticeable feature is the grouping of the lines, in the more refrangible end of the spectrum, into pairs, in which the broader and more conspicuous lines are separated by an interval of about six degrees, while to their right, at a distance of about one degree, another but much feebler line in each case is seen. In the spectrum of cupric iodide no such symmetrical arrangement is evident. With plumbic chloride the same extension of the lines into the upper end of the spectrum takes place. Many of the bands in the spectra of the plumbic salts are beautifully shaded, and commence with a feeble illumination on the side toward the less and increase to a line of maximum brightness on the side toward the more refrangible end of the spectrum, where they abruptly terminate.

Without detailing in this place, what takes place when the various metallic compounds are examined, it will be interesting to note briefly the deportment of one of them-cupric chloride. When a mass of this salt, which has not previously been freed from water of crystallization, is heated on a platinum wire in the flame of a Bunsen burner, it imparts in the first place a greenish illumination to a large portion of the flame. On examining the flame through dark blue glass, it is seen that the part immediately above the heated substance is of a deep blue color. This becomes tinged with violet, and later a tongue of reddish flame rises in the centre of the blue. If the substance be pushed into the hotter part of the burner, this flame changes to a bright white light, which at its upper edge becomes lurid again. The spectrum in this case is continuous throughout the middle and lower portion, the separate bands of violet still remaining distinct.

It is evident that we have been studying phenomena of a mixed character. When we carefully heat a mass of this salt, so that it is slowly volatilized along with aqueous vapor at the outer edge of the flame, a green band, extending from 60.8 to 72.4 , alone makes its appearance. If we heat a concentrated solution of cupric chloride, the red lines from $37.8-44.8$ appear synchronously with the green from $60 \cdot 8$ to $72 \cdot 4$. It is only at higher temperatures that the great number of lines in the blue and violet make their appearance, and it is not until the salt is fused that the spectrum becomes con. tinuous. We do not, in this case, attribute the continuity of the spectrum to the diffusion of incandescent particles of the solid sub. stance throughout the flame, but to the widening out of the bands 
in every part of the spectrum until their fusion produces white light.

At a future time we hope to replace this preliminary essay by more carefully prepared drawings, and by a more extended table of the lines referred in position to normal wave-lengths, and in inten. sity to the solar spectrum taken as a standard.

\title{
ABSOLUTE SYSTEM OF ELECTRICAL MEASUREMENTS.
}

\author{
By Josiah P. CoOkr, JR. \\ (Continued from page 140.)
}

These two forces would form a couple acting with a leverage equal to one-half the length of the needle, so long as the needle remains in the meridian. Hence the effect of the couple would be represented by $\frac{\mathrm{CL} m l}{k_{2}}$, where $m l$ represents the magnetic moment.

When deflected to an angle $d$ the force would act as strongly as ever, but the effect of the couple on the needle would be dimin. ished because the moment is not now $m l$, but $m l$ cos. $d$.

The strength of the couple now, is therefore-

$$
\frac{\mathrm{c} \mathrm{L} m l}{k_{2}} \cos . d \text {. }
$$

When the needle comes to equilibrium the effect of this couple is exactly balanced by a second couple created by the earth's magnetism. The space

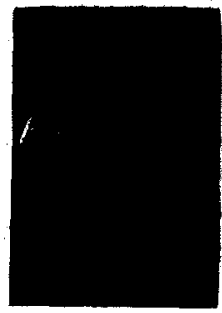
in which our experiments are made is a magnetic field of uniform intensity, the direction of the forces in that field are sensibly par. allel, and the intensity of that field is capable of measurement. The directions of the lines of force are shown by a dipping needle, but as a magnetic needle is usually hung so that it oan only move in a horizontal plane, it is usual to estimate only the horizontal force of the earth's magnetism, which we will represent by H. $^{*}$ The effect of the earth's magnetism on a pole would be $m$ H, and the effioat of the magnetic couple on our needle when pointing $\mathrm{E}-\mathrm{W}$, would be

* The mean horizontal component for 1862 at Kew, in England, was 1.7592 units, i.e., a unit pole weighing 1 gramme, and free to move in a horizontal plane would acquire under the earth's magnetism a velocity of 1.7592 metres a second. 